\title{
NEW PERSPECTIVES OF POINT CLOUDS COLOR MANAGEMENT THE DEVELOPMENT OF TOOL IN MATLAB FOR APPLICATIONS IN CULTURAL HERITAGE
}

\author{
M. Pepe ${ }^{a}, *$, S. Ackermann, L. Fregonese, C. Achille \\ ${ }^{a}$ Polytechnic of Milan, 20133, Milan, Italy - (massimiliano.pepe, sebastiano.ackermann, luigi.fregonese, cristiana.achille)@ polimi.it
}

\author{
Commission II
}

KEY WORDS: Color Models, Cultural Heritage, Terrestrial Laser Scanner, Photogrammetry, Colored Point Clouds Integration

\begin{abstract}
:
The paper describes a method for Point Clouds Color management and Integration obtained from Terrestrial Laser Scanner (TLS) and Image Based (IB) survey techniques. Especially in the Cultural Heritage (CH) environment, methods and techniques to improve the color quality of Point Clouds have a key role because a homogenous texture brings to a more accurate reconstruction of the investigated object and to a more pleasant perception of the color object as well. A color management method for point clouds can be useful in case of single data set acquired by TLS or IB technique as well as in case of chromatic heterogeneity resulting by merging different datasets. The latter condition can occur when the scans are acquired in different moments of the same day or when scans of the same object are performed in a period of weeks or months, and consequently with a different environment/lighting condition. In this paper, a procedure to balance the point cloud color in order to uniform the different data sets, to improve the chromatic quality and to highlight further details will be presented and discussed.
\end{abstract}

\section{INTRODUCTION}

Nowadays, modern survey techniques, such as Close-Range Photogrammetry or Laser Scanner (terrestrial and aerial) (Eisenbeiss et al, 2000; Pepe et al., 2016) due to their capability to produce three-dimensional point clouds in a fast as well as accurate mode, are widely employed in many field, such as cultural heritage, architecture, 3D documentation and mapping (Remondino, 2011).

Laser scanner is usually competitive for indoors surveys (e.g. mining, tunneling, etc.) allowing to perform textured scans even in poor light conditions but keeping mostly a uniform color brightness with the right illumination (natural and/or artificial) once the scans are edited and joined to obtain the final 3D model. On the other hand, the quality of outdoors scans in terms of texture are often influenced by the exterior light situation: unless we have a diffused or natural cloudy light condition during the entire acquisition session, the constant presence of sun and shadows could have a negative impact on the final result.

Digital photogrammetry is a much flexible technique that allows to reach better results in terms of appearance (texture) of the $3 \mathrm{D}$ models in less time (depending on the object complexity), thanks to a usually higher resolution of the images employed to compute the model texture, compared to the panoramic ones normally used to texturize the laser scanner clouds. In addition, the points composing the clouds obtained with image matching based algorithms are usually colored by reading and averaging the RGB values of the pixels that generated the specific point of the cloud, compared to the laser scanner technique where each scan is colored separately, with no color averaging for the neighboring scans.

However, the image-based approach can run into some limitation when big and/or complex objects must be acquired to obtain accurate and high resolution models in case of indoors surveys. In such cases, a much more attention during the acquisition step must be kept, especially regarding the backlit and a reduced depth of field; in addition, extra equipment, such as tripods, spotlights, etc., could be also required, considerably extending the acquisition and the editing time as well.

Beyond the geometrical information, the structure of Point Clouds files usually contains the color information that follows the RGB model. For example, laser scanner cloud files usually are registered by following the $X Y Z I R G B$ format, where $X Y Z$ represent the spatial coordinates, $I$ the intensity and $R G B$ the color. One of the most common file format is the ASCII one, an "open" format file which is compatible with almost all commercial software. At any rate, such file structure is actually considered far from being "optimized". To overcome this limitation, a much more suitable file format for laser data storage is the LAS one (Lidar Exchange Format), a binary and public file format with precise specifications about its digital structure approved by the American Society for Photogrammetry and Remote Sensing (ASPRS): initially created for storage of lidar data, it became a standard even for photogrammetric point clouds. This file format is widely employed by several software developers in Matlab environment (Samberg, 2002; Korzeniowska et al., 2012; Wang et al. 2011; Pepe et al, 2015).

Beyond the RGB model, different color models have been developed (Ibraheem, et al 2012); two common color models are usually employed: the Hue-Saturation-Value (HSV) and Hue-Saturation-Lightness (HSL) (Smith et al, 1996) created by Alfred H. Munsell in the early 1900s. HSV and HSL models are particularly useful because they describe the colors in a way closer to the human perception (Platanioti et al, 2000; Pepe et al., 2016). This models are represented by using the cylindrical coordinate system, where the Hue is the angle around the cylinder axis, the Saturation is the distance from the axis while the Value/Lightness is the distance along the axis. Hue represents the color perception (red, yellow, green, blue, purple)

\footnotetext{
* Corresponding author: massimiliano.pepe@ polimi.it
} 
and varies from $0^{\circ}-360^{\circ}$, where $0^{\circ}$ corresponds to the Red, $120^{\circ}$ to the Green and $240^{\circ}$ to the Blue. The Saturation represents the "purity" of the color and varies from $0 \%$ to $100 \%$ : by decreasing the Saturation, color appears more pale. The highest value represents the full saturation $(100 \%)$, while the minimum $(0 \%)$ is equivalent to the grayscale. The Value and Lightness are the quality by which we distinguish a light color from a dark one (Munsell, 1905) and its value varies from 0\% to $100 \%$. By considering the cylindrical coordinate system and by fixing a Lightness value, Hue and Saturation are equivalent to the polar coordinates of any point in the plane (Bourke, 2016). The chosen model in the present work for color management is the HSL. The following equations show the mathematical relationships that allow the passage from the RGB system to the HSL one (Brivio, 2006):

$$
\left\{\begin{array}{l}
H=\frac{1}{2 \pi} \operatorname{arctg}\left(\frac{2 R-G-B}{\sqrt{3(G-B)}}\right) \\
S=\sqrt{R^{2}+G^{2}+B^{2}-R G-R B-B G} \\
L=\frac{1}{3}(R+G+B)
\end{array}\right.
$$

A detailed description of the algorithm for the transition from HSL to RGB is described in the paper of Smith (1978), while the algorithm that allows the transformation from RGB to HSL used in this work is due to Fishkin (1990).

\section{MATLB ALGORITHM AND FUNCTION TO CHANGE THE COLOR OF POINT CLOUDS}

\subsection{Functions for editing of the Point Clouds}

One way to characterize the color of a colored point clouds is through its histogram, as is the case in digital images. The histogram of a colored point cloud is a graph in which is represented the frequency of occurrence of each gray level for each color channel, i.e. the number of times that a certain value is repeated in the point cloud.

Some transformations in the spatial domain allow to suitably modify the histogram in order to improve the visualization. This transformation applies individually to each point of the cloud, and can be described according to:

$\checkmark$ intensity (or gray) level of the point (point operations);

$\checkmark$ spatial position (geometric operations).

In the first case (used in the present work) since the transformation operates on the gray levels and not on the spatial positions, it is possible to adopt the following relation (Gonzalez et al., 2002):

$$
[y]=[\tau] \cdot[x]
$$

where the elements of the modified vector $[y]$ can assume values in the dynamic ranges related to the employed color model ( $R G B$ or $H S L)$.

The color transformation of the points can be obtained by using linear and non-linear functions. The following equation (3) is an example of linear transformation:

$$
y=a \cdot x
$$

In the equation (3), if a>1 there is an expansion of the dynamic range, while if $a<1$ an histogram compression is obtained. It is important to underline that an excessive histogram expansion can lead to a saturation condition as in the processing step the values admitted for $[y]$ must results within the admitted values of the chosen color model (e.g. $0 \leq R \leq 255$ ).

\subsection{Workflow}

The goal of the proposed developed algorithm is to improve the color quality of a portion or of a complete Point Cloud. By considering the raw data it is possible to obtain a new Point Clouds corrected by following the selected correction values of the texture.

By applying linear functions it is possible modify the single values of the RGB or HSL model. In the case of modification of the HSL model parameters, a double transformation is required. However, the application of these functions on the first Point Clouds can be irreversible, due to the fact that it is not possible to obtain the original date set with a simple inverse operation. The color adjustment procedure is iteratively repeated until an enough desired texture for the entire model is reached.

The graphical flowchart of the methodology applied in this paper is resumed in Fig. 1.

In addition, specific functions have been implemented in order to avoid inconsistent values of the color both in the RGB that HSL model

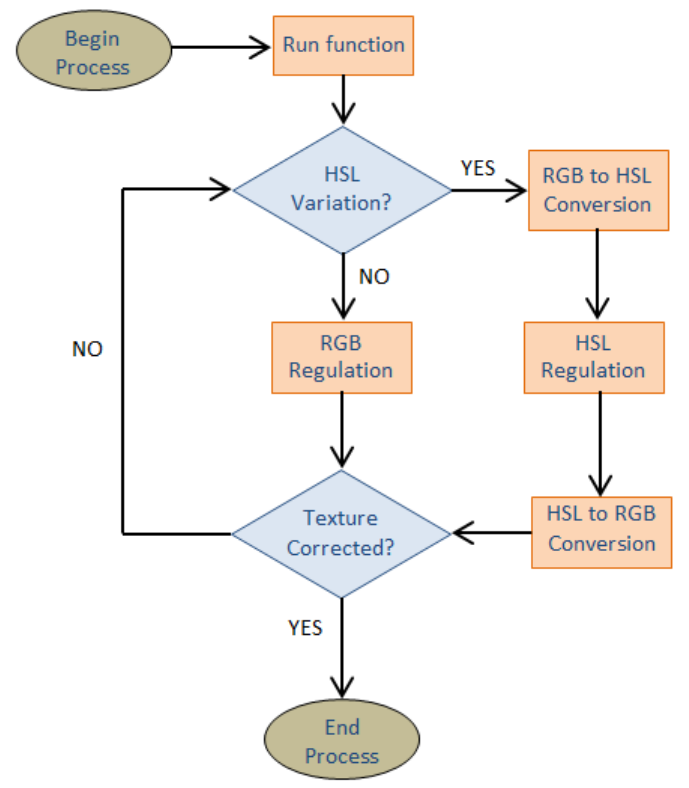

Figure 1. Workflow of the algorithm

\section{CASE STUDY}

In order to show the efficacy of this method, a certain number of application cases will be described below. In particular, the first case studio concerns the improvement of an individual data sets obtained through Close Range Photogrammetry (CRP) technique. The second application case is the survey of a portion of the surrounding defensive wall of Castel Nuovo in Naples: due to the dimension of the wall and to the 
organizational necessities for the progress of the construction activities (the wall has been found during the construction of the underground station "Municipio" in Naples), the survey has been performed in two different acquisition session between 2014 and 2015. In the latter case, the goal is the homogenization of the separated datasets acquired by terrestrial laser scanner. A third case study regards the integration of two data sets: the first obtained with TLS acquisition technique, and the second with CRP one.

\subsection{Color enhancement of a single dataset: the ship in relief on the rock}

The first case study is the survey of a ship in relief sculpted on a rock in the archeological area of Lindos (Rhodes island Greece). This relief is located at the base of the acropolis on which is located the Sanctuary of Athena. The survey has been carried out by employing a Pentax K-x DSLR camera with a 18$55 \mathrm{~mm}$ f/3.5-5.6 lens: a total amount of 54 images have been captured and then processed with Photoscan software in order to extract a dense Point cloud of about 28 million points. Due to a massive presence of natural shadows, the captured photos were slightly underexposed (figure 2-left) and an image overexposure was not taken into consideration to avoid nosed images. Therefore, using the developed tool, it was possible to optimize the color quality of the point cloud (figure 2-right). In the regulation step of the color, a prior analysis of the histograms can result useful to decide the correction parameters. In the Figure 2, the histograms related to the red, green, blue, hue, saturation and lightness for the two point clouds (before and after the color correction) are reported.
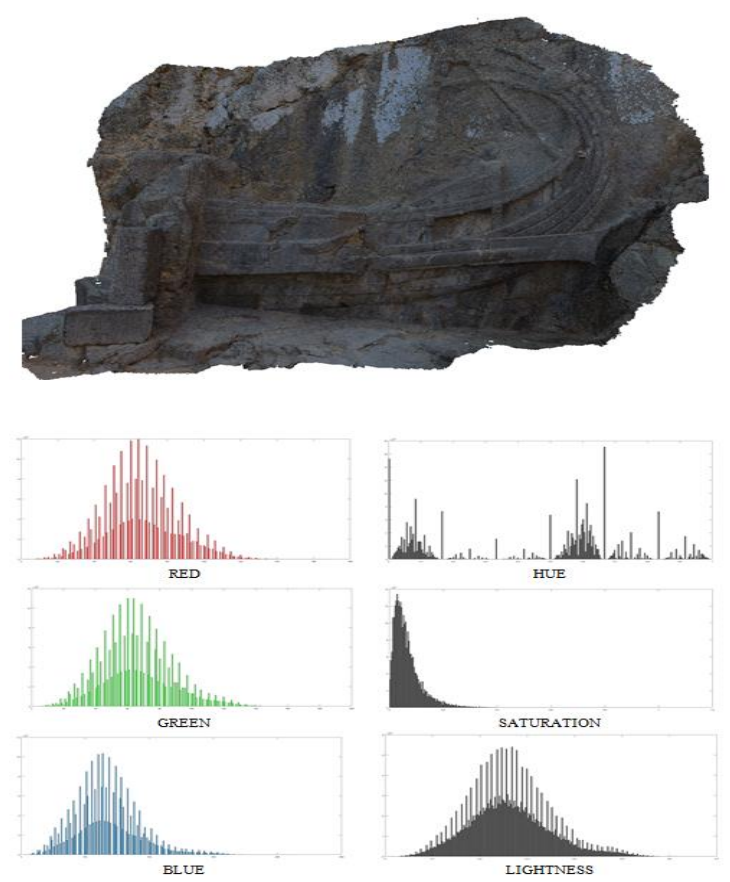

The correction functions applied to the Point Clouds and their relative value in order to achieve this improvement of color, as shown in the following table 1 :

\begin{tabular}{|l|c|}
\hline Color correction function & value \\
\hline Red & $+3 \%$ \\
Green & $+2 \%$ \\
Blue & $-2 \%$ \\
Hue & $0 \%$ \\
Saturation & $-3 \%$ \\
Lightness & $+30 \%$ \\
\hline
\end{tabular}

Table 1 - Parameters correction of photogrammetry Point clouds

\subsection{Color enhancement and integration of multi dataset: the case of the surrounding defensive wall of "Castel Nuovo"}

The second case study concerns the color improvement of two datasets acquired with a Leica HDS7000 phase-based laser scanner with a resolution of $6.3 \mathrm{~mm} @ 10 \mathrm{~m}$ and textured by using a Canon 5D Mark II DSLR camera equipped with a $15 \mathrm{~mm}$ fisheye lens.

A total amount of 14 textured scans ( 8 scans for the left block (figure 3-left) and 6 scans for the right block (figure 3-right)) were performed, allowing to obtain a final cloud of about a hundred million of points.

The point clouds processing has been performed with Leica HDS Cyclone software.
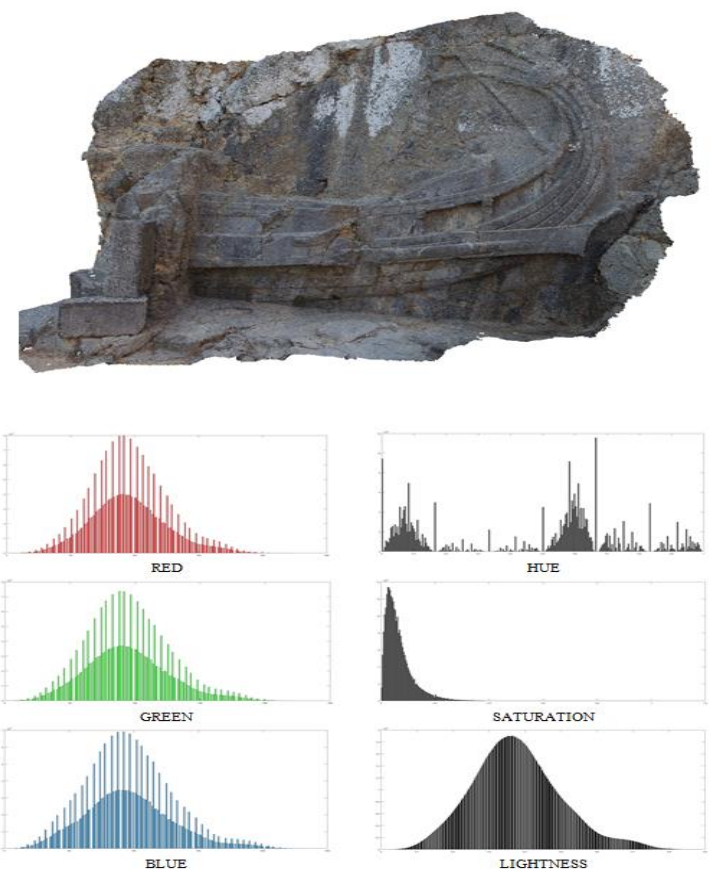

Figure 2. Raw Point Clouds data (left) - Point Clouds after the color correction (right)

In Figure 3 is it possible to observe that the two datasets have a dissimilar texture: the different natural illumination on the wall during the acquisition step has influenced the final texture result. Indeed, the dataset on the left has been acquired with a weather condition of partially shadowed, while the dataset on the right has been acquired in a different day with a condition of the wall totally exposed to the sunlight.

By applying the proposed algorithm, the contrast between one cloud and the neighbor ones has been sensibly reduced and the transition between the two cloud blocks appears smoother than 
in the original condition, increasing the homogeneity of the whole point clouds (Figure 3).

In particular, it has been substantially correct the luminosity of left block through its increasing, while on the right-hand block a reduction of the saturation and luminosity has been set up. All the correction parameters adopted for this case are resumed in the Table 2.

The Figure 3 shows the situation of the two clouds before and above the color optimization, allowing to better understand the final result.

\begin{tabular}{|c|c|c|}
\hline \multirow{2}{*}{$\begin{array}{c}\text { Color correction } \\
\text { function }\end{array}$} & \multicolumn{2}{c|}{ Surveyed part } \\
\cline { 2 - 3 } & Left & Right \\
\hline Red & $-2 \%$ & $+5 \%$ \\
Green & $0 \%$ & $0 \%$ \\
Blue & $0 \%$ & $+2 \%$ \\
Hue & $+2 \%$ & $+3 \%$ \\
Saturation & $0 \%$ & $-40 \%$ \\
Lightness & $+30 \%$ & $-20 \%$ \\
\hline
\end{tabular}

Table 2 -Parameters correction of TLS Point clouds
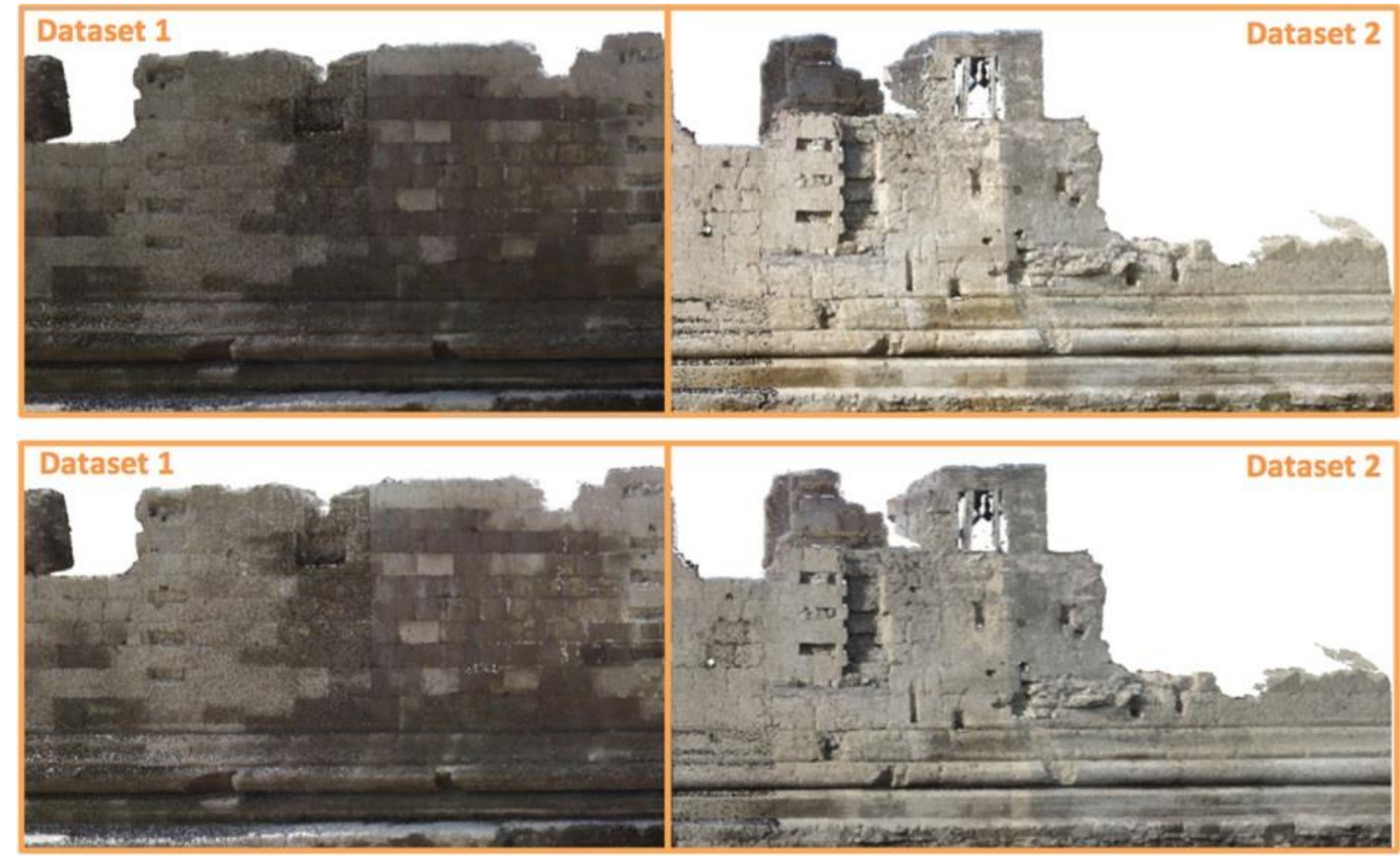

Figure 3. Point cloud raw (above) - Point Cloud after adjustment (below)

\subsection{Integration of TLS and CRP datasets}

A particular survey has been conducted to document an historical wall founded during the excavation of a station shaft of the new line 6 in Naples (Italy). This wall was located in a position surrounded on three sides by concrete bulkheads built before the excavation started. The important height (approximately $6 \mathrm{~m}$ from the base of the wall) of the bulkheads and their proximity to the wall to be surveyed led to poor lighting and planning difficulties for the data acquisition: for this reason, an acquisition in two step with two different survey technology has been preferred for this specific case. The terrestrial laser scanner has been chosen in order to collect data in less time with a better accuracy for the vertical parts of the wall: a total amount of 22 textured scans with a resolution of $6.3 \mathrm{~mm} @ 10 \mathrm{~m}$ have been performed for the vertical parts of the walls. However, the physical limitations of the area made impossible to obtain a perfect cloud without data gaps. Indeed, there were considerable difficulties in acquiring the part of the top of the wall, due to its particular height that made impossible to place the instruments on the top and/or to operate with enough safety.

To overcome this situation, a photogrammetric survey limited to these uncompleted portions has been preferred by employing a wearable small photographic crane, in order to capture images with a shutter time of $1 / 2$ second and to keep a good depth of field at the same time. This method allowed to perform both nadiral and tilted images to better compute the final cloud around the corners of the wall. A DSLR Canon EOS 5D Mark II camera, equipped with a $24 \mathrm{~mm}$ f/2.8 fixed lens, has been employed to perform the photogrammetric survey. The complete 3D colored point cloud of the top section of the wall has been obtained by processing the acquired 573 photos in Photoscan.

However, the photogrammetric point cloud appears much clearer than the one obtained with TLS: the post-processing of both the cloud block by using the developed algorithm, permitted to correct the point clouds color differences and to sensibly improve the homogeneity of the two different data sets, completely recovering the original TLS cloud gaps. In this case, the vertical part of the wall it has been brighten up while the upper part has been darkened. Even if two different acquisition 
technique has been employed, the only parameter that needed to be modified was the lightness. The final point cloud, is shown in Figure 4.

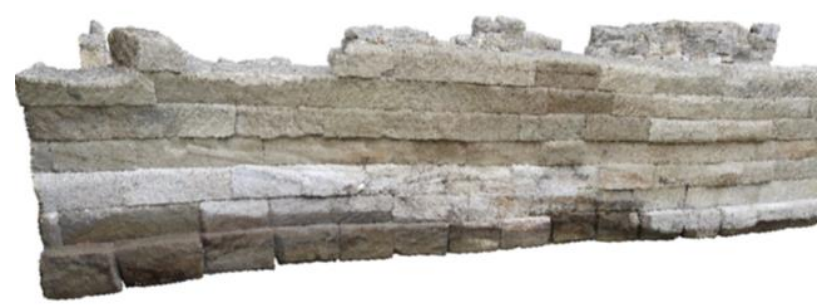

Figure 4. Final Point Clouds after the color correction

\section{CONCLUSIONS}

The developed algorithm above described permitted to sensibly improve the texture quality of point cloud models. Indeed, as reported in the discussed cases studies, the chromatic correction applied on the individual data sets allowed to obtain a much homogenous and uniform point cloud. In addition, in those cases where there is the necessity to merge data sets obtained from different techniques or emphasize some particular detail, the color quality improvement of the point clouds leads to a better result as well.

Even if the tests were performed on archaeological artifacts, the method could be also adapted and used in other fields in which the appearance quality of is a not negligible aspect other than the geometric accuracy.

\section{REFERENCES}

Eisenbeiss H., L. Zhang, 2006. Comparison of DSMs generated from mini UAV imagery and terrestrial laser scanner in a cultural heritage application. In: The International Archives of Photogrammetry, Remote Sensing and Spatial Information Sciences, Dresden, Germany, Vol. XXXVI, Part 5, pp. 90-96.

Ford, A., \& Roberts, A., 1998. Colour space conversions. Westminster University, London, pp. 1-31.

Gonzalez, R. C., \& Woods, R. E., 2002. Digital image processing.

Ibraheem, N. A., Hasan, M. M., Khan, R. Z., Mishra, P. K., 2012. Understanding color models: a review. ARPN Journal of Science and Technology, 2(3), pp. 265-275.

Korzeniowska, K., 2012. Modelling of water surface topography on the Digital Elevation Models using LiDAR data, in AGILE'2012 International Conference on Geographic Information Science, Avignon, April, 24-27 , 2012, pp. 333335 .

Pepe M., Prezioso, G., 2015. A Matlab Geodetic Software for Processing Airborne LIDAR Bathymetry Data. In: The International Archives of the Photogrammetry, Remote Sensing and Spatial Information Sciences, Piano di Sorrento, Italy, Vol. XL-5/W5, pp. 167-170.

Pepe, M., Prezioso G., 2016. Two Approaches for Dense DSM Generation from Aerial Digital Oblique Camera System. In: Proceedings of the 2nd International Conference on
Geographical Information Systems Theory, Applications and Management, pp. 63-70.

Pepe, M., Ackermann, S., Fregonese, L., Achille, C., 2016. 3D Point Cloud Model Color Adjustment by Combining Terrestrial Laser Scanner and Close Range Photogrammetry Datasets. World Academy of Science, Engineering and Technology, International Journal of Computer, Electrical, Automation, Control and Information Engineering, 10(11), pp. 1840-1846.

Remondino, F., 2011. Heritage recording and 3D modeling with photogrammetry and 3D scanning. Remote Sensing, 3(6), pp. 1104-1138.

Samberg, A., 2007. An implementation of the ASPRS LAS standard. In: The International Archives of the Photogrammetry and Remote Sensing, Espoo, Finland, Vol. XXXVI, Part 3/W52, pp. 363-372.

Smith A. R., Lyons E. R., 1996. HWB-A more intuitive huebased color model. Journal of Graphics Tools, 1(1), pp. 3-17.

Smith, A. R., 1978. Color gamut transform pairs. ACM Siggraph Computer Graphics, 12(3), pp. 12-19.

Wang, X., Zhou, K., Yang, J., and Lu, Y., 2011. MATLAB tools for LIDAR data conversion, visualization, and processing. In: Proceedings of SPIE - The International Society for Optical Engineering, Vol. 8286, pp. 1-8. 